\title{
The New Extended KdV Equation for the Case of an Uneven Bottom
}

\author{
Anna Karczewska ${ }^{1}$, Piotr Rozmej ${ }^{2}$ \\ ${ }^{1}$ Faculty of Mathematics, Computer Science and Econometrics \\ University of Zielona Góra, Szafrana 4a, 65-246 Zielona Góra, Poland \\ E-mail: A.Karczewska@wmie.uz.zgora.pl \\ ${ }^{2}$ Faculty of Physics and Astronomy \\ University of Zielona Góra, Szafrana 4a, 65-246 Zielona Góra, Poland \\ E-mail:P.Rozmej@if.uz.zgora.pl
}

Received: 20 November 2018; revised: 03 December 2018; accepted: 04 December 2018; published online: 20 December 2018

\begin{abstract}
The consistent derivation of the extended $\mathrm{KdV}$ equation for an uneven bottom for the case of $\alpha=O(\beta)$ and $\delta=O\left(\beta^{2}\right)$ is presented. This is the only one case when second order KdV type nonlinear wave equation can be derived for arbitrary bounded bottom function.
\end{abstract}

Key words: Surface gravity waves, nonlinear equations, second order perturbation approach, uneven bottom

\section{INTRODUCTION}

Korteweg and de Vries have discovered surface gravity nonlinear waves [3] more than 120 years ago in the first order perturbation approach. Next, in 1990, Marchant and Smyth obtained the extended $K d V$ equation (also called $K d V 2$ ) in the second order approach. Before our and our co-workers' papers $[1,2]$ these equations were obtained only for the case of the flat bottom. In these papers, in addition to standard small parameters $\alpha=\frac{a}{h}$ and $\beta=\left(\frac{h}{l}\right)^{2}$ we introduced the third one defined as $\delta=\frac{a_{h}}{h}$. In these definitions $a$ denotes the wave amplitude, $h$ the average water depth, $l$ the average wavelength and $a_{h}$ the amplitude of the bottom variations. We considered the case of $\alpha=O(\beta)$ and $\delta=O(\beta)$, that is, when all three small parameters are of the same order. Then, with standard assumptions for incompressible, inviscid fluid and irrotational motion, we applied the second-order perturbation approach to the set of Eulerian equations. This set, written in nondimensional variables has the following form (see, e.g., Eqs. (2)-(5) in [2])

$$
\begin{aligned}
\beta \phi_{x x}+\phi_{z z} & =0 \\
\eta_{t}+\alpha \phi_{x} \eta_{x}-\frac{1}{\beta} \phi_{z} & =0, \text { for } z=1+\alpha \eta \\
\phi_{t}+\frac{1}{2} \alpha \phi_{x}^{2}+\frac{1}{2} \frac{\alpha}{\beta} \phi_{z}^{2}+\eta & =0, \text { for } z=1+\alpha \eta \\
\phi_{z}-\beta \delta\left(h_{x} \phi_{x}\right) & =0, \text { for } z=\delta h(x) .
\end{aligned}
$$

Equation (1) is the Laplace equation valid for the whole volume of the fluid. Equations (2) and (3) are so-called kinematic and dynamic boundary conditions at the surface, respectively. The equation (4) represents the boundary condition at the non-flat bottom. For abbreviation all subscripts denote the partial derivatives with respect to particular variables, i.e. $\phi_{t} \equiv \frac{\partial \phi}{\partial t}, \eta_{n x} \equiv \frac{\partial^{n} \eta}{\partial x^{n}}$ and so on.

For the flat bottom, the boundary condition at the bottom is $\phi_{z}=0$. In this case, the perturbation approach of the first 
order with respect to small parameters leads to the famous Korteweg-de Vries equation [3]

$$
\eta_{t}+\eta_{x}+\alpha \frac{3}{2} \eta \eta_{x}+\beta \frac{1}{6} \eta_{3 x}=0 .
$$

The second order perturbation approach leads to the $e x$ tended $K d V$ equation of the form [4]

$$
\begin{aligned}
\eta_{t}+\eta_{x} & +\alpha \frac{3}{2} \eta \eta_{x}+\beta \frac{1}{6} \eta_{3 x}+\alpha^{2}\left(-\frac{3}{8} \eta^{2} \eta_{x}\right) \\
& +\alpha \beta\left(\frac{23}{24} \eta_{x} \eta_{2 x}+\frac{5}{12} \eta \eta_{3 x}\right)+\beta^{2} \frac{19}{360} \eta_{5 x}=0 .
\end{aligned}
$$

In $[1,2]$ we tried to extend the second-order approach to the case $\delta \neq 0$, that is, the case of a non-flat bottom.

Recently, it was pointed out in [5] that one step in our derivation performed in $[1,2]$ and leading to the KdV2 equation for an uneven bottom was inconsistent, and therefore the derived equation [2, Eq. (18)] is not correct. We derived our equation [2, Eq. (18)] in good faith. However, using different notations for small parameters $\alpha, \beta, \delta$ we did not recognize the proper order of terms related to the bottom function.

In [6] we presented the proof that for the case $\alpha=O(\beta)$ and $\delta=O(\beta)$ a $\mathrm{KdV}$ type wave equation exists neither in the first nor in higher orders. This happens because the set of the Boussinesq equation resulting from (1)-(4) can not be made compatible for arbitrary shape of the bottom function when $\alpha=O(\beta)$ and $\delta=O(\beta)$.

In this short note we focus on the only one case when the nonlinear wave equation of $\mathrm{KdV}$ type, describing unidirectional waves, can be derived in a consistent second order approach for an arbitrary shape of the bottom function. This is done in Section II.. Next, in Section III. we present some examples of the motion of KdV2 solitons over the uneven bottom in the form of piece-wise linear functions.

\section{DERIVATION OF THE NONLINEAR WAVE EQUATION FOR THE CASE OF $\alpha=O(\beta)$ AND $\delta=O\left(\beta^{2}\right)$}

In [5], the author points out that the consistent second order perturbation approach can be achieved when all small parameters are related to only one, assuming for the considered case

$$
\alpha=A \beta, \quad \delta=D \beta^{2} .
$$

In the standard approach the velocity potential is assumed in the form of the series

$$
\phi(x, z, t)=\sum_{m=0}^{\infty} z^{m} \phi^{(m)}(x, t) .
$$

For the flat bottom case ( $\delta=q=0$ ) equations (1) and (4) allow us to express all $\phi^{(m)}(x, t)$ with even $m$ only, by $f(x, t):=\phi^{(0)}(x, t)$ and its even $x$-derivatives. For the uneven bottom case, to satisfy the equation (4), the velocity potential has to contain also odd $m$ terms. In general, the velocity potential fulfilling Laplace equation can be expressed in the following form

$$
\begin{aligned}
\phi(x, z, t) & =\sum_{m=0}^{\infty} \frac{(-1)^{m} \beta^{m}}{(2 m) !} \frac{\partial^{2 m} \phi^{(0)}}{\partial x^{2 m}} z^{2 m} \\
& +\sum_{m=0}^{\infty} \frac{(-1)^{m} \beta^{m+1}}{(2 m+1) !} \frac{\partial^{2 m+1} \phi^{(1)}}{\partial x^{2 m+1}} z^{2 m+1},
\end{aligned}
$$

where $\phi^{(1)}=\phi^{(1)}(x, t)$. The explicit form of this velocity potential is

$$
\begin{aligned}
\phi & =f^{(0)}-\frac{1}{2} \beta z^{2} f_{2 x}^{(0)}+\frac{1}{24} \beta^{2} z^{4} f_{4 x}^{(0)}-\frac{1}{720} \beta^{3} z^{6} f_{6 x}^{(0)}+\cdots \\
& +\beta z F-\frac{1}{6} \beta^{2} z^{3} F_{2 x}+\frac{1}{120} \beta^{3} z^{5} F_{4 x}+\cdots
\end{aligned}
$$

where we denote $f=\phi^{(0)}$ and $F=\phi_{x}^{(1)}$.

Now, we insert the general form of velocity potential (9) into the bottom boundary condition which in this case is

$$
\phi_{z}-D \beta^{3}\left(h_{x} \phi_{x}\right)=0, \quad \text { for } \quad z=D \beta^{2} h(x)
$$

obtaining relation

$$
\begin{gathered}
F-D \beta^{2}\left(h f_{x}\right)_{x}-\frac{1}{2} D^{2} \beta^{5}\left(h^{2} F_{x}\right)_{x}+\frac{1}{6} D^{3} \beta^{7}\left(h^{3} f_{3 x}\right)_{x} \\
+\frac{1}{24} D^{4} \beta^{10}\left(h^{4} F_{3 x}\right)_{x}-\frac{1}{120} D^{5} \beta^{12}\left(h^{5} f_{5 x}\right)_{x}+\cdots=0 .
\end{gathered}
$$

Then, since we are interested in second order equations, we can neglect all terms except the first one and use

$$
F=D \beta^{2}\left(h f_{x}\right)_{x}
$$

which inserted into (9) gives the velocity potential in the form

$\phi=f-\frac{1}{2} \beta z^{2} f_{2 x}+\frac{1}{24} \beta^{2} z^{4} f_{4 x}-\frac{1}{720} \beta^{3} z^{6} f_{6 x}+\cdots$

$+D \beta^{3} z\left(h f_{x}\right)_{x}-\frac{1}{6} D \beta^{4} z^{3}\left(h f_{x}\right)_{3 x}+\frac{1}{120} D \beta^{5} z^{5}\left(h f_{x}\right)_{5 x}+\cdots$

Inserting velocity potential (13) into (2) and (3) and retaining terms up to the second order one obtains the set of the Boussinesq equations in the following form (as usual $\left.w=f_{x}\right)$ 


$$
\begin{aligned}
& \eta_{t}+w_{x}+\beta\left(A(\eta w)_{x}-\frac{1}{6} w_{3 x}\right) \\
&+ \beta^{2}\left(-A \frac{1}{2}\left(\eta w_{2 x}\right)_{x}+\frac{1}{120} w_{5 x}-D(h w)_{x}\right)=0 \\
& w_{t}+\eta_{x}+\beta\left(A w w_{x}-\frac{1}{2} w_{2 x t}\right) \\
&+\beta^{2}\left(-A\left(\eta w_{x t}\right)_{x}+A \frac{1}{2} w_{x} w_{2 x}-A \frac{1}{2} w w_{3 x}\right. \\
&\left.\quad+\frac{1}{24} w_{4 x t}\right)=0
\end{aligned}
$$

In the first order this system reduces to the common $\mathrm{KdV}$ system, with

$$
w=\eta+\beta\left(-A \frac{1}{4} \eta^{2}+\frac{1}{3} \eta_{2 x}\right)
$$

which ensures the KdV equation

$$
\eta_{t}+\eta_{x}+\beta\left(A \frac{3}{2} \eta \eta_{x}+\frac{1}{6} \eta_{3 x}\right)=0
$$

Now, we aim to satisfy the Boussinesq system (14)-(15) with the terms of the second order included. So, we set

$$
w=\eta+\beta\left(-A \frac{1}{4} \eta^{2}+\frac{1}{3} \eta_{2 x}\right)+\beta^{2} Q .
$$

Then we insert the trial function (18) into (14) and (15) and retain terms up to second order in $\beta$. This yields the set of two equations

$$
\begin{aligned}
\eta_{t} & +\eta_{x}+\beta\left(A \frac{3}{2} \eta \eta_{x}+\frac{1}{6} \eta_{3 x}\right)+\beta^{2}\left(-A^{2} \frac{3}{4} \eta^{2} \eta_{x}\right. \\
+ & \left.\frac{1}{12} \eta_{x} \eta_{2 x}-\frac{1}{12} \eta \eta_{3 x}+\frac{17}{360} \eta_{5 x}-D(h \eta)_{x}+Q_{x}\right)=0
\end{aligned}
$$

and

$$
\begin{aligned}
\eta_{t}+\eta_{x} & +\beta\left(-A \frac{1}{2} \eta \eta_{t}+A \eta \eta_{x}-\frac{1}{6} \eta_{2 x t}\right) \\
+\beta^{2} & \left(-A^{2} \frac{3}{4} \eta^{2} \eta_{x}-A \frac{1}{2} \eta_{x} \eta_{x t}+A \frac{1}{4} \eta_{t} \eta_{2 x}+A \frac{5}{6} \eta_{x} \eta_{2 x}\right. \\
& \left.-A \frac{3}{4} \eta \eta_{2 x t}-A \frac{1}{6} \eta \eta_{3 x}-\frac{1}{8} \eta_{4 x t}+Q_{t}\right)=0
\end{aligned}
$$

Now, we subtract the equation (20) from (19). This gives

$$
\begin{aligned}
& \beta\left(A \frac{1}{2} \eta\left(\eta_{t}+\eta_{x}\right)+\frac{1}{6}\left(\eta_{2 x t}-\eta_{3 x}\right)\right) \\
& +\beta^{2}\left[A\left(\frac{1}{2} \eta_{x} \eta_{x t}-\frac{1}{4} \eta_{t} \eta_{2 x}-\frac{3}{4} \eta_{x} \eta_{2 x}+\frac{3}{4} \eta \eta_{2 x t}+\frac{1}{12} \eta \eta_{3 x}\right)\right. \\
& \left.+\frac{1}{8} \eta_{4 x t}-\frac{17}{360} \eta_{5 x}+Q_{x}-Q_{t}-D(h \eta)_{x}\right]=0
\end{aligned}
$$

.

In (21), in order to replace $t$-derivatives by $x$-derivatives we use $Q_{t}=-Q_{x}$ and the properties of the first order equation (17), that is, $\eta_{t}=-\eta_{x}-\beta\left(A \frac{3}{2} \eta \eta_{x}+\frac{1}{6} \eta_{3 x}\right)$, again retaining only terms up to second order. Solving the result with respect to $Q_{x}$ and integrating over $x$ we find

$$
Q=\frac{1}{2} D(h \eta)+A^{2} \frac{1}{8} \eta^{3}+A \frac{3}{16} \eta_{x}^{2}+A \frac{1}{2} \eta \eta_{2 x}+\frac{1}{10} \eta_{4 x}
$$

This form of the correction function makes the Boussinesq system (14)-(15) compatible and allows to the derive explicit form for the wave equation for the case of $\alpha=O(\beta)$ and $\delta=O\left(\beta^{2}\right)$. Finally, we have

$$
\begin{aligned}
w=\eta & +\beta\left(-A \frac{1}{4} \eta^{2}+\frac{1}{3} \eta_{2 x}\right)+\beta^{2}\left(\frac{1}{2} D(h \eta)\right. \\
& \left.+A^{2} \frac{1}{8} \eta^{3}+A \frac{3}{16} \eta_{x}^{2}+A \frac{1}{2} \eta \eta_{2 x}+\frac{1}{10} \eta_{4 x}\right)
\end{aligned}
$$

and

$$
\begin{aligned}
\eta_{t} & +\eta_{x}+\beta\left(A \frac{3}{2} \eta \eta_{x}+\frac{1}{6} \eta_{3 x}\right)+\beta^{2}\left(-\frac{1}{2} D(h \eta)_{x}\right. \\
- & \left.A^{2} \frac{3}{8} \eta^{2} \eta_{x}+A \frac{23}{24} \eta_{x} \eta_{2 x}+A \frac{5}{12} \eta_{x} \eta_{3 x}+\frac{19}{360} \eta_{5 x}\right)=0
\end{aligned}
$$

The equation (24) is the nonlinear wave equation, for uneven bottom, when $\alpha=O(\beta), \delta=O\left(\beta^{2}\right)$, derived consistently within the second order perturbation approach.

Since $\delta=D \beta^{2}$ we can come back to original notations for small parameters, used in [2]. Then equations (23) and (24) take the following forms

$$
\begin{aligned}
w=\eta & -\frac{1}{4} \alpha \eta^{2}+\frac{1}{3} \beta \eta_{2 x}+\delta \frac{1}{2} h \eta \\
& +\frac{1}{8} \alpha^{2} \eta^{3}+\alpha \beta\left(\frac{3}{16} \eta_{x}^{2}+\frac{1}{2} \eta \eta_{2 x}\right)+\frac{1}{10} \beta^{2} \eta_{4 x}
\end{aligned}
$$

and

$$
\begin{aligned}
\eta_{t}+\eta_{x} & +\frac{3}{2} \alpha \eta \eta_{x}+\frac{1}{6} \beta \eta_{3 x}-\delta \frac{1}{2}(h \eta)_{x}-\frac{3}{8} \alpha^{2} \eta^{2} \eta_{x} \\
& +\alpha \beta\left(\frac{23}{24} \eta_{x}^{2}+\frac{5}{12} \eta \eta_{2 x}\right)+\beta^{2}\left(\frac{19}{360} \eta_{5 x}\right)=0
\end{aligned}
$$

These forms of equations (25) and (26) may be misleading, since the terms with $\delta$, looking as first order ones, are, in fact, of second order.

The equation (26), limited to the case $\delta=D=0$, is the extended $K d V$ equation or $K d V 2$ [4]. This equation is nonintegrable. Despite this fact, we found several forms of analytic solutions to KdV2: soliton solutions in [2], cnoidal solutions $\left(\sim \mathrm{cn}^{2}\right)$ in [7] and superposition cnoidal solutions $\left(\sim \mathrm{dn}^{2} \pm \sqrt{m} \mathrm{cn} d n\right)$ in [8,9], see the monograph [11], as well. 
The equation (26) is the second order wave equation directly taking into account bottom variation derived consitently for the case of $\alpha=O(\beta)$ and $\delta=O\left(\beta^{2}\right)$.

The wave equation (26) is very similar to the not fully correct [2, Eq. (18)]. The latter contains, apart from the leading term from the bottom $-\frac{1}{2} \delta(h \eta)_{x}$, two other terms which resulted from not fully consistent derivation.

\section{NUMERICAL SIMULATIONS}

In this section, we tentatively examine the motion of solitons, solutions of the KdV2 equation (6), entering the region where the bottom is no longer even. In these tests, we use our numerical code based on the finite difference method. The code was described in detail in [2] and [6].

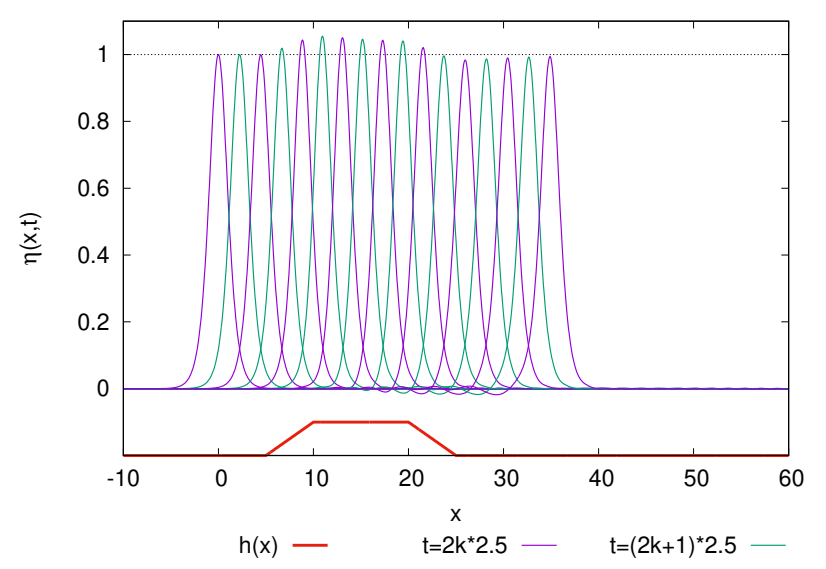

Fig. 1. Time evolution of the KdV2 soliton moving over the trapezoidal bump, $t \in[0,30]$. The thick red line shows the shape of the bottom function $h(x)$ (not in scale)

Since the equation for the uneven bottom (26) differs from the extended $\mathrm{KdV}$ equation (6) by only single term $-\frac{1}{2} \delta(h \eta)_{x}$ then for initial conditions we can choose the single soliton solution of (6) for soliton position far from the obstacle. Such single soliton solutions were found by us in $[2$, Sect. V]. Below we present numerical evolution of such soliton when $\alpha=\beta=0.2424$ and $\delta=2 \beta^{2} \approx 0.1175$. For $\alpha \approx 0.2424$ the amplitude of such soliton is one. Then the initial condition is $\eta(x, t=0)=\operatorname{sech}^{2}(\sqrt{0.599} x)$.

Distortions of the soliton shape caused by interaction with uneven bottom observed in Figs. 1 and 2 are small. The main effect of this interaction is the increase of the wave amplitude during the motion over the obstacle with a simultaneous decrease of the wave velocity. These effects for the motion of KdV2 solitons over localized obstacle were predicted in approximate analytic solutions found by us in [10].

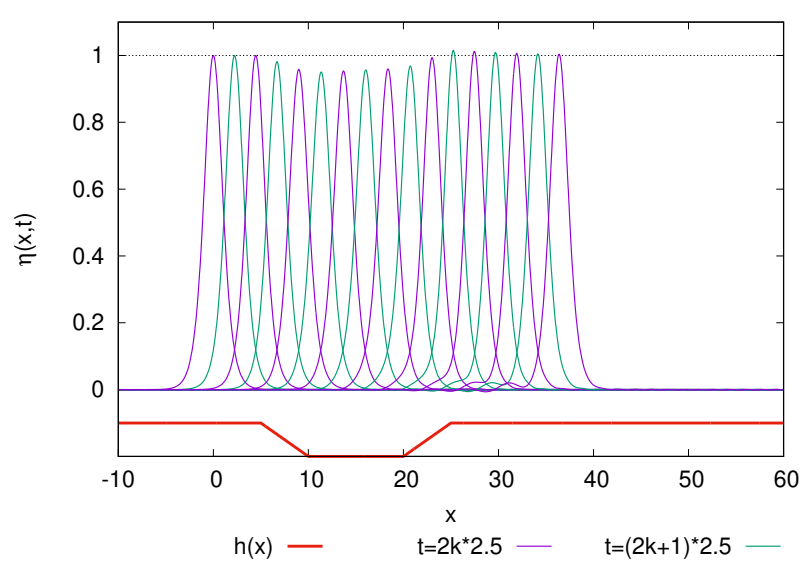

Fig. 2. Time evolution of the KdV2 soliton moving over the trapezoidal well, $t \in[0,30]$. The thick red line shows the shape of the bottom function $h(x)$ (not in scale)

Comparison of the numerical evolution of KdV2 solitons obtained with the equation (26) with that resulted from the not fully correct equation [2, Eq. (18)] shows an important difference. The radiation of small amplitude wavetrain in front of the main wave, present in evolution according to [2, Eq. (18)] seems to dissappear in evolution according to the equation (26) displayed in Figs. 1 and 2.

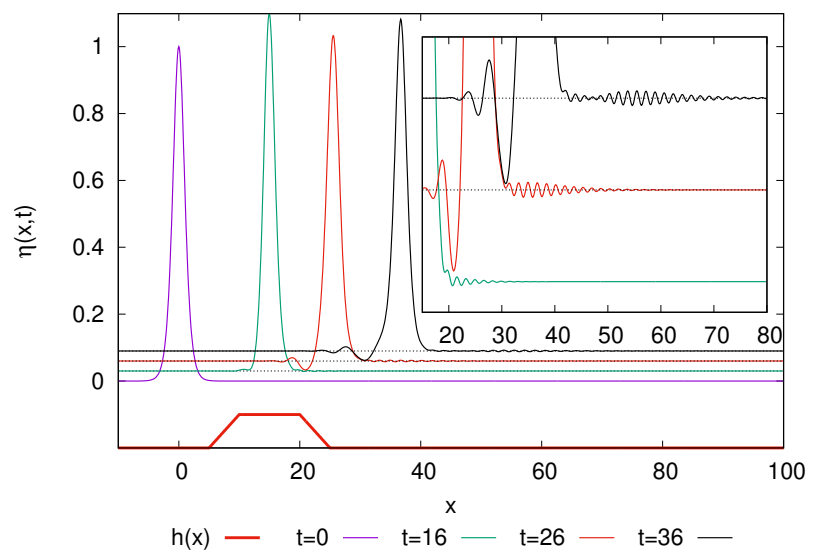

Fig. 3. Several profiles of the KdV2 soliton moving over the trapezoidal well. In the inset the radiation of the faster wave packets of small amplitude is clearly seen

The thorough inspection of the calculated data reveals that this radiation still exists, but with much smaller amplitude (in Figs. 1 and 2 this amplitude is comparable to the linewidth). In order to enhance these effects we performed additional calculations in which we set $\alpha=\beta=0.2424$ and $\delta=3 \beta^{2} \approx 0.176$. Several profiles of the wave obtained in the numerical evolution of KdV2 soliton according to the equation (26) are displayed in Fig. 3. The creation and then 
detachment of the small amplitude wave packet in front of the main wave is clearly exposed in the inset. This is qualitatively the same feature as observed in our previous papers $[1,2,10]$ for wave motion according to the not fully correct equation [2, Eq. (18)]. Quantitatively the effect has much smaller amplitude, for realistic values of parameters $\alpha, \beta, \delta$ it is smaller than $1 \%$ of the solitons amplitude. On the other hand, even such small effect suggests the origin of the very tiny wrinkles observed always on the water surface at the seashore.

We are sure that this is the real effect, not an artifact of numerical simulation. Since our code utilizes periodic boundary conditions we performed calculations on much wider $x$-interval than displayed in figures above. In such cases, when the soliton moves far from the end of the $x$ interval, the boundary conditions do not influence the shape of the localized wave.

\section{Acknowledgment}

We thank the author of [5] for his detailed explanation of the consistent perturbation approach in the case of several small parameters. His remarks allowed us to derive the correct second order wave equation explicitly containing terms from the uneven bottom, for the arbitrary, bounded bottom function.

\section{References}

[1] A. Karczewska, P. Rozmej, Ł. Rutkowski, A new nonlinear equation in the shallow water wave problem, Physica Scripta,
89, 054026, (2014).

[2] A. Karczewska, P. Rozmej, E. Infeld Shallow water soliton dynamics beyond KdV, Physical Review E, 90, 012907, (2014).

[3] D.J. Korteweg, G. de Vries, On the change ofform of the long waves advancing in a rectangular canal, and on a new type of stationary waves, Phil. Mag. (5), 39, 422 (1895).

[4] T.R. Marchant, N.F. Smyth, The extended Korteweg-de Vries equation and the resonant flow of a fluid over topography, Journal of Fluid Mechanics, 221, 263-288, (1990).

[5] The review by the anonymous referee of the paper: P. Rozmej, A. Karczewska, Comment on the paper "The third-order perturbed Korteweg-de Vries equation for shallow water waves with a non-flat bottom" by M. T.C. Fokou Kofané, A. Mohamadou and E. Yomba, Eur. Phys. J. Plus, 132, 410 (2017), arXiv:1804.01940.

[6] P. Rozmej, A. Karczewska, Extended KdV equation for the case of uneven bottom, arXiv:1810.07183. Submitted to Phys. Rev. E.

[7] E. Infeld, A. Karczewska, G. Rowlands, P. Rozmej, Exact cnoidal solutions of the extended KdV equation, Acta Phys. Pol. A, 133, 1191-1199, (2018).

[8] P. Rozmej, A. Karczewska, E. Infeld: Superposition solutions to the extended KdV equation for water surface waves, Nonlinear Dynamics 91, 1085-1093, (2018).

[9] P. Rozmej, A. Karczewska, New Exact Superposition Solutions to KdV2 Equation, Advances in Mathematical Physics. 2018, Article ID 5095482, 1-9, (2018).

[10] G. Rowlands, P. Rozmej, E. Infeld, A. Karczewska, Single soliton solution to the extended KdV equation over uneven depth, Eur. Phys. J. E, 40, 100, (2017).

[11] A. Karczewska, P. Rozmej, Shallow water waves - extended Kortewed-de Vries equations, University of Zielona Góra, 2018.

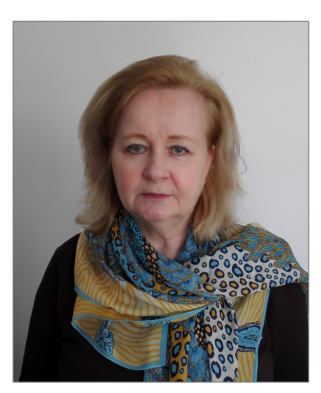

Anna Karczewska is a professor at the University of Zielona Góra. She holds an MSc degree from the Warsaw Technical University, a PhD degree from Jagiellonian University, Cracow, and habilitation from the University of Zielona Góra. Her fields of interest include deterministic and stochastic integral equations, in particular Volterra equations of integral and fractional order, fluid dynamics and Korteweg-de Vries type equations. Together with P. Rozmej she has recently published a monograph titled "Shallow water waves extended Korteweg-de Vries equations".

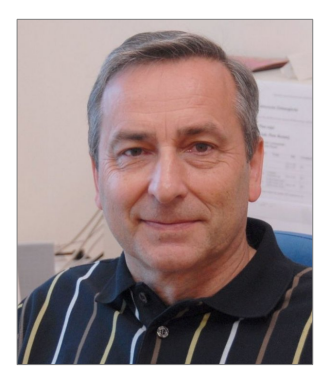

Piotr Rozmej is a full professor at the Faculty of Physics and Astronomy, University of Zielona Góra, and head of the Division of Mathematical Methods in Physics. He holds an MSc Degree from Maria CurieSkłodowska University (UMCS), Lublin, a PhD degree from Warsaw University, and habilitation from UMCS. He became a professor in 1992 and full professor in 1997. He has spent several years working as a senior researcher at the Joint Institute for Nuclear Research (Dubna, USSR), Gesellschaft für Schwerionenforschung (Darmstadt, Germany) and Institut des Sciences Nucléaires (Grenoble, France). He has worked in several fields of theoretical nuclear physics like nuclear structure, nuclear reactions, properties of heavy and superheavy nuclei. Other areas of his scientific activity include quantum optics, in particular wave packet dynamics, deterministic chaos, nonlinear dynamics, solitons, numerical modeling and simulations. 